\title{
Espacios virtuales universitarios
}

\author{
Joaquín García, Ferrán Ferrer, \\ Rafael Mompó, Luis M. Naya.
}

\section{Introducción}

La documentación disponible sobre el tema es cada vez más abundante. Pero por documentos, debemos hoy entender tanto los textos escritos como cualquier otro "producto" (ni siquiera tienen aún un nombre definido) que transfiere información significativa (textos o secuencias de acciones) en cualquier otro soporte (CD, WWW, INTERNET...). Su apreciación como documento o como informe de actividad de investigación, dentro de la comunidad científica es aún muy imperfecto. Publicar un texto, que informa sobre una innovación educativa, puede ser considerado un trabajo científico, pero editar un $\mathrm{CD}$ que contiene el desarrollo de la innovación podría no ser considerado un documento.

En este trabajo, tomaremos el punto de vista de hacerlo desde la perspectiva de una reflexión teórica, poniendo en común lo que consideremos - a partir de la propia experiencia reflexiva algunos temas mayores teóricos y prácticos del nuevo contexto comunicacional planteado por la generalización de las aplicaciones y el uso de las TIC (Nuevas Tecnologías de la Información y la Comunicación), para la reflexión-acción pedagógica.

Nuestro trabajo tiene dos partes bien diferenciadas: la primera, un análisis esquemático del nuevo contexto creado por las TIC; la segunda, tomar un producto de información universitaria como fuente de indicios de la introducción de las TIC en la actividad de formación dentro de la Universidad.

\section{El contexto creado por las TIC}

Quizás el primer punto a poner de relieve consista en subrayar la raíz de la innovación tecnológica. En nuestro criterio la innovación fundamental se encuentra en el descubrimiento y aplicación a la comunicación de un nuevo soporte: el soporte electrónico. En la perspectiva más común tanto la imprenta como la cibernética se analizan como instrumentos de comunicación, técnicas de soporte de la comunicación y de transporte del significado. Lo cual incita a tomar en consideración el tema, p.e. del libro, y de las interfaces tecnológicas de su producción, por el camino en el que las tecnologías implicadas se asocian a la palabra (el problema de los contenidos de los libros, la animación a la lectura, el planteamiento crítico y reflexivo del lector...). El mensaje y la palabra asociada al mismo a través de los signos escritos, son los que proporcionan únicamente el significado y el valor cultural; como si reflexionar sobre la imprenta, p.e., debiera únicamente coincidir con la reflexión sobre el texto que con ella se imprime. Paralelamente han sido muchos los estudiosos que han puesto de relieve la importancia, p.e. del libro como objeto material, sujeto de expresión artística y vehículo de cultura [1], o la importancia de la imprenta como factor de desarrollo y como mediador de la introducción de nuevos modos de discurso, nuevas maneras de ser Autor y Actor social [2]. Pero, muchas veces, este papel concedido al desarrollo de las tecnologías que aplican y desarrollan un soporte de la información (el papel) y a los modos comunicacionales, organizacionales de la interacción que hacen posible queda un poco oculto.

Cabe, sin embargo, otra manera de plantear el problema, sin invalidar la más frecuente, sino 
complementándola. Toda forma de cultura requiere de mediadores que preserven la experiencia acumulada y se constituyan , a su vez, en mediadores de transmisión cultural, en instrumentos para los procesos sociales de transmisión cultural [3]. La palabra sería el instrumento primigenio de preservación-transmisión de experiencia, la escritura sería un segundo sistema que da origen a las culturas lectoescritoras, consideramos que las Tecnologías de la Información y la Comunicación (TIC) constituyen la más contemporánea mediación con este objetivo, aunque no sea la única aplicación posible. En el primer caso el relato en todas sus formas (conversacional, narrativa, lírica, dramática...) constituye la mediación en la acción comunicativa, en el segundo el texto en todas sus interfaces hasta nuestros libros, en el tercero la hipertextualidad como punto de referencia. Unas se van asumiendo en las siguientes y al tiempo mantienen sus específicas formas de construcción de discurso.

Si en vez de considerar la cultura como un patrimonio de sucesos, hechos y productos culturales, tomamos el punto de vista del intercambio simbólico, entonces la cultura presenta la figura de un sistema clausurado de intercambios simbólicos, un sistema clausurado de conversaciones, un sistema de coordinaciones de relaciones entre actores del significado. Lo denominamos clausurado porque el contexto que crea la cultura determina parcialmente, al menos, zonas de comprensión y de familiaridad con las expresiones y las acciones al tiempo que limita y cierra, parcialmente al menos, la comprensión y la familiaridad respecto al exterior, al diferente, al extraño. Pues bien, las posibilidades de la expresión y de la acción de comunicación cambian sustancialmente con el cambio en los soportes de la comunicación: cambian los roles de enunciación (roles de actor comunicante), los roles de recepción y cambia también la situación de comunicación.

Las necesidades de los actores en las culturas de oralidad se resuelven mediante los recursos propios de la modalidad expresiva que permite la palabra: motivación en el tema, relevancia de sus partes, recursos para la memorización, integración con otros componentes culturales, espacio y límites de la situación de comunicación, incluso la estructura y el contenido posible de la comunicación...etc. Todo ello termina componiendo, cuando se torna reflexivo, la Retórica, con todos sus recursos de persuasión, estrategias de comprensión, métodos de favorecimiento de la retención, sistemas de argumentación...etc.

El libro como mediador permite otras formas de discurso: la linealidad argumentativa, la sistemática del pensamiento, el argumento narrativo...De todo ello se ha beneficiado la posibilidad de constituir instituciones sociales, como los códigos legales, los archivos de propiedad, las bibliotecas, incluso la ortodoxia de las religiones y su pretensión de universalidad. Las TIC permiten la conectividad intertextual, la accesibilidad dirigida, la composición abierta del texto, la integración de la imagen y el sonido...En estos dos últimos casos, cambian igualmente los roles de autor de la comunicación, el concepto y la modalidad de la distancia comunicacional: las relaciones entre el lector y el texto del autor, entre el sujeto (no hay nombre, solemos decir audiencia) y los "textos" multimedia.

No sólo quedan afectadas por la modalidad de mediación las acciones comunicativas, sino que también las formas de organización social y los sistemas simbólicos. Se posibilitan cambios en los sistemas sociales porque se inserta la mediación dentro de los objetivos de la acción social haciendo posibles otros modos sociales de comunciación y de organización, otros roles sociales (cultura escriba frente a cultura del noble guerrero o cazador). Muchos autores afirman que la Polis es un modo social asociado a la escritura. Pero también los sistemas simbólicos cambian: las religiones de proyección universal se encuentran asociadas al libro inspirado, surge el dogma y la ortodoxia. Frente a la tradición aparece la ley [4]. 
La técnica, no es meramente un medio sino un mensaje tanto hacia fuera sobre la concepción del mundo, como hacia dentro del sujeto respecto al modo y oportunidad de estar en la situación, modifica el modo de vida y la percepción de la calidad de vida posible. La escritura lo demostró. Esto lleva a que las relaciones entre acciones de formación y tecnología no se reducen a las aplicaciones de la tecnología [5], ya que promueven también conformaciones de la subjetividad respecto a sus modos de discurso. Un estudio de Antropología educativa no se detiene en los límites habituales de la Antropología cultural: la cultura patrimonial; lleva las cosas un poco más lejos interesándose por la cultura de uso por parte de las comunidades de sujetos y no meramente por la cultura objetiva de que dispone como patrimonio del grupo. Ruth Benedict, por tomar un ejemplo, entiende que la Antropología es en definitiva la ciencia de la costumbre [6]. Tal es el sentido profundo de que el medio es el mensaje. Pero, más que el rasgo patrimonial el elemento significativo de la cultura estriba en "el individuo viviendo en su cultura, y a la cultura como vivida por los individuos". De ahí la razón contenida en la palabra cultura cuando se considera lo que tiene de "cultivo". La cultura en el sentido más literal es proceso y mediación social de acceder a la cultura objetiva. Entonces se comprende que las mediaciones no son meros soportes de la transmisión sino que contienen parte de su sentido: el hombre es una especie que tiene la "costumbre" de hablar, la "costumbre de escribir" y, ahora, está adquiriendo la costumbre de "multimediar" la acción comunicativa y de "navegar" en un hiperespacio de comunicación. En los dos primeros sistemas de comunicación se vio la oportunidad de introducir el rol de "maestro", de profesor; en el tercero, todavía los maestros se encuentran fuera y, lo que es más preocupante, todavía las acciones de formación miran desde fuera esta oportunidad creada por las TIC.

Según esto el objetivo primario de la formación básica es enseñar calidad en la palabra y enseñar calidad en la lectura. Palabra en la propia lengua y en otras lenguas, lectura en la propia lengua y en otras lenguas, lectura de otras formas expresivas que no son lingüísticas. En el contexto actual terminará por constituir un objetivo básico la conversión del sujeto en usuario de esta nueva forma de mediación comunicacional, por permitir el acceso a formas culturales de las que, de otra manera, quedaría marginado. Recordemos que la introducción de la escritura creó, como efecto asociado, la marginación del analfabeto; aún desconocemos los efectos socioculturales de la marginación de las TIC, tanto en el campo institucional, como en el empresarial, como en el subjetivo. De momento, únicamente tenemos evidencias de los posibles efectos de un empleo sin objetivos de formación. Observamos los efectos controlados de su empleo en la gestión económica y administrativa, pero tenemos poca información sobre los efectos sobre la forma de pensamiento, sobre la inteligencia y la comprensión con éxito; en el fondo, porque ya se han introducido sin posibilidad de marcha atrás en las acciones económicas y administrativas de los Sistemas Sociales. Todavía no se puede afirmar que se hayan introducido en el corazón de los Sistemas de Enseñanza. Las gentes dan por hecho que constituye una nueva herramienta y una nueva oportunidad para trabajar, pero todavía no se ha generalizado como instrumento de formación. Tenemos cultura de la palabra, tenemos cultura lectoescritora, cosa que demostramos por la habilidad, la calidad del uso, la orientación de las decisiones... etc; pero se carece de la cultura correspondiente del uso y el empleo de las TIC. Del mismo modo que se dio con anterioridad la especialidad del "escriba" y del "calígrafo", todavía el uso de las TIC se encuentra muy especializado. Se da, sin embargo, una diferencia sustancial en el último caso. Mientras que, cualquiera de nosotros puede ser "autor" de oralidad y autor de "texto", una prueba es este artículo, difícilmente se puede ser autor de un producto TIC, en muchos casos sin asociarse en equipos productores de "textos multimedia", por la dificultad que entraña a un tiempo ser hábil en la programación, en el diseño y en el contenido informativo de ese tipo de productos.

Siguiendo a Castells, este nuevo paradigma cultural se caracteriza por:

- La información es su materia prima, se trata de NT que actúan sobre la información y no sólo de información que afecta al desarrollo tecnológico; 
- Omnipresencia en la actividad humana dentro del Sistema Social de las consecuencias de esta mediación técnológica;

- Profundización de una "lógica en red" de las utilizaciones de estas NT, morfología en red de la configuración topológica de la actividad que promueven;

- Cambios profundos en las categorías desde las que pensamos todos los procesos de la práctica humana y que en los años 80 se inició desde la perspectiva que proporcionó en ciencias sociales el desarrollo de las "teoría del caos" y que en los años 90 se extiende como perspectiva de "complejidad", como un nuevo modo de considerar la diversidad [7].

\section{Los contextos virtuales}

La filosofía tradicional, ha estudiado principalmente los procesos de transformación que van del posible al real y del virtual al actual. "Virtual" en la filosofía clásica es existencia en potencia, que tiende a convertirse en existencia en acto (como el modo de estar contenido el árbol en la semilla), o potencia (virtud) que tiende a la acción. En este sentido "virtual" se contrapone a real, como formas diferentes de existencia [8].

El proceso actual, al menos en el léxico, propone la tarea de reflexión sobre la transformaciòn inversa, desde el real y el actual hacia el virtual. Esta es la propuesta de la "simulación", como método para la comprensión, incluso como argumento para la explicación.

En el lenguaje coloquial la referencia a virtual queda asociada a irreal. Es el "pasillo" cibernético construido por una empresa en la película "Acoso". Frente a ello estaría lo real en lo tangible, lo virtual en la ilusión.

El campo de lo virtual es el espacio de llegada de un camino inverso que lleva desde lo real y actual, con todas sus posibilidades, a la construcción de una entidad diferente [9]. Supone encontrar en lo real y lo actual principios y reglas de interacción entre componentes y crear con ellos un nuevo espacio de funcionamiento en el que se sustituyen las circunstancias reales (en el espacio y tiempo actuales) por circunstancias propuestas a voluntad por los actores o seleccionadas de entre un catálogo programado en el que la voluntad o la aleatoriedad practica las selecciones. De ahí la utilidad del concepto de simulación para este tipo de construcciones.

Este éxodo ("hors-là”), según lo denomina Michel Serres [10], se posibilita también dentro de las funciones de la memoria (huir al recuerdo) o dentro de las funciones de la imaginación (huir al fantasma) o en las del conocimiento (huir a la teoría, la cual es, por supuesto, el meollo de la simulación). Así planteadas las cosas los vectores de lo real son la territorialidad, la actualidad temporal, la situación circunstancial; los vectores del espacio virtual suponen la ruptura de la unidad temporal, de la proximidad espacial, y de la situación; crea una oportunidad de acción en un espacio de entidad diferente, en un tiempo que no es mi historia (aunque consuma tiempo), y transforma esa acción en situación para el comportamiento individual. El desarrollo de la virtualización ya se advierte en construcciones como el espacio mitológico (recuérdese que contiene un tiempo con retorno eterno), o en las construcciones literarias que llevó a cabo la cultura del texto. Aunque Jerome Bruner lo denominara "mundos posibles", ontológicamente se trata de mundos virtuales [11].

La virtualización a la que hacemos referencia en el nuevo léxico que aparece con las TIC dice relación, por un lado al desacoplamiento de las personas respecto a sus espacios de referencia particulares y, por otro, a la posibilidad de simular el funcionamiento de sistemas reales o crear el funcionamiento de sistemas imaginarios. Pero haciendo frecuente referencia al concepto "virtual", no podemos olvidar que uno de los efectos reales de las TIC consiste 
precisamente en la potenciación (multiplicación y expansión) del sistema de comunicación interhumana real.

Además de las evidencias anteriores sobre tiempo, espacio, circunstancia, se dan otros caracteres que deben ser tomados en consideración.

La aceleración de los procesos de todo tipo, de las prácticas en la cultura, en los que se encuentra implicada la comunicación; y, con ello, la aceleración de la acción.

La fractalización del espacio de referencia, del sitio para la comunicación, el pensamiento y para la práctica... y, con ello, la recomposición profunda de toda clase de identidades: la identidad de actor en la comunicación (agd@gugu.usal.es), del puesto de trabajo y de las relaciones de producción (tutor virtual), de lugar (site-Web), del ir a la institución (universidad virtual...), tele-trabajo.

La virtualización, por lo tanto, ya se introduce, frente a la realidad cuando la contamos mediante la palabra o la narramos en el texto, como cuando construimos una simulación. En todos los casos se cumple el principio de la diferencia entre la realidad y el "texto"(hablado o escrito).

\section{La virtualidad en el texto y el hipertexto}

Si el símbolo de la escritura es el libro y su tecnología la de la impresión-reproducción de los libros, el símbolo de las TIC en relación con los procesos de formación es el hipertexto. La idea de hipertexto procede de Vannevar Bush en 1945 [12], el término hipertexto es de Theodore Nelson que añade a los procesos dialogales y a los de lectura por secuencia la idea de escritura-lectura no lineal y los siguientes principios o potenciales de acción:

- Reconstructividad permanente del texto, porque el texto queda permanentemente abierto a construcción-reconstrucción.

- Heterogeneidad de los componentes de información, porque permite su soporte inclusión de letras, toda clase de grafismos y grafos, imágenes, sonidos.

- Reticularidad de configuración, y no meramente secuencialidad. La encapsulación de la información tiene una modalidad fractal en el sentido de que permite desde cada nudo informacional acceder por multitud de caminos a la totalidad de la información.

- Unificación por activación, en el sentido de que no posee una unidad interna como el libro, sino que la unidad de secuencia depende de la activación del hipertexto depende de la activación desde el exterior, tanto respecto al uso como respecto a la composición. El hipertexto permite indefinidas maneras de transformarse en texto.

- Organización topológica o por nexos de la información, conectividad, su uso debe someterse a la estructura de los nexos porque no constituye un espacio homogéneo. El hipertexto no puede ser ojeado, sino navegado.

- Descentración de la información como consecuencia de la indefinida ramificación posible. Ni tiene primera ni última página y su índice es imposible.

El hipertexto se acomoda mejor, simula mejor, el modo de proceder del entendimiento humano en el que tiene prioridad la reticularidad sobre la secuencia. El proyecto hipertexto más que proyectar un libro proyecta la noción de simulación de textos, bibliotecas, procesos, mecanismos, sistemas en general. 
Entre el diálogo, el texto y el hipertexto se da un reempleo de interfaces de la comunicación. En el diálogo, p.e., se daban las interfases tema de conversación que permitía organizar el contexto y los significados, la interfase pregunta-respuesta...

Casi no advertimos la importancia de las interfases que introduce el libro y que reproduce el operador-reproductor de la imprenta: cabeceros de capítulo, párrafos, índices, paginación, notas de pie de página, referencias cruzadas... permiten lecturas sectoriales, consultas,... y otras interfases materiales que ya han sido aludidas. La democratización del libro no es posible sin la presencia de todas estas interfases, incluidas las del tamaño y el peso. Lo mismo ocurre con el sistema biblioteca, con sus interfases depósito, ordenamiento, catálogo, fichas bibliográficas; incluso la posibilidad de "hojear".

El plegamiento de la información en el hipertexto es indefinido, con muy poca superficie de texto inmediatamente accesible y con una posibilidad de manipulación caótica. Frente a la limitación de acceso-superficie, por pantalla, (no obstante el cursor) se encuentra la posibilidad instantánea de acceso a no importa que punto de información previamente indexado. De ahí que junto al proceso de lectura aparezca otro de naturaleza cualitativa diferente que se denomina hoy navegación por el texto.

El hipertexto permite su asociación a sistemas expertos que posibiliten de manera interactiva la construcción por parte del usuario de diagramas de significados con lo que el usuario no solamente navega por la información sino que construye y organiza la información que personalmente va adquiriendo.

No planteo si el hipertexto suprimirá el libro. De momento tenemos que afirmar que nunca se ha impreso tanto papel como en los países desarrollados que extienden la informática. Como tampoco suprimió la calidad y validez formativa del diálogo la presencia del libro , por más que un libro es una invitación permanente al aprendizaje en la soledad y el aislamiento individualista.

Hemos dado la fecha de 1945 para la idea de hipertexto. En ese momento el sueño era la posibilidad informática de la Biblioteca de Alejandría. En la Silicon Valley de California Steve Jobs y Steve Wozniac construyen las primeras decenas de ejemplares de lo que constituía el "no va mas" del bricolaje: el ordenador personal. En 1975 Bill Gates y Paul Allen lanzan el Basic. Entre los slogans de Berkeley se encontraba el de "los ordenadores para el pueblo". Se reproduce un movimiento análogo al que duro decenas de siglos por el que la escritura pasó de los círculos sagrados de los templos, o de los nobles servicios administrativos, a la popularización del libro por las imprentas. Se pasó de los secretos dominios de los ejércitos, o de las sofisticadas gestiones de las grandes empresas al computador personal. Sólo que ahora estamos en un período que generosamente podemos estimas en cincuenta años. Durante este tiempo las interfases que se han incorporado a los ordenadores en su mayor parte han sido de respuesta al usuario, para terminar siendo un instrumento tan simple como una máquina de escribir y una mesa de despacho virtual.

Cuando en 1984 aparece Macintosh de Apple se puede decir que se convierte el proyecto de la Biblioteca de Alejandría en un proyecto de uso personal: el hipertexto personal, el cual redescubre el valor del icono como ideograma de comunicación lectoescritora.

Paralelamente a la historia del hipertexto, Douglas Engelbart, del Augmentation Research Center de la Universidad de Stanford sueña con las redes de trabajo cooperativo, que P. Lévy denomina "collecticiels". Lo cual obligaba a la coherencia de interfases lo que permite de un lado la comunicación operativa - muchos pueden intervenir en las mismas cosas- y la coherencia de la habilidad, tras cada habilidad adquirida se facilita la adquisición de la siguiente, pone el acento en la comunicación de los sistemas cognitivos humanos mediados por interfases cada vez más simples, más sensoriomotrices. Por este camino de dulcificación 
de la relación hombre-instrumento es por el que el ordenador ha ido pasando de máquina para la automatización de los cálculos, a tecnología para la actividad intelectual de los grupos humanos. Con el mismo cerebro los grupos consiguen acrecentar globalmente la gama de la actividad -su espectro-, la cantidad y la complejidad de la actividad. El ordenador esta constituyendo progresivamente un contexto, un nicho ecológico de actividad mental. La atención no se centra tanto en el diseño de la máquina cuando en la configuración de los proyectos, las TIC se han revelado muy versátiles para la respuesta.

\section{Espacio virtual de la formación en el trabajo universitario}

Como hace 5.000 años ocurrió con la escritura, las TIC en la Universidad ha entrado con fuerza y de manera irreversible a través de la gestión, de la manipulación y el control de las operaciones con datos: gestión económica , gestión de datos administrativos en centralización creciente.

Las acciones de formación se encuentran todavía en proceso de crecimiento por focos de acción aislada. La reflexión no se debe orientar por la vía de la apología de la telepresencia, sino por la vía de la utilización reflexiva de este nuevo sistema de mediación para la realización de actividades de formación. Lo que fundamentalmente debe cambiar es el modo de llevar a cabo el trabajo pedagógico; ello supone cambio en el trabajador y cambio en los modos de llevarlo a cabo. Como lo que no ha cambiado es el hombre, ni su aparato mental, ni sus necesidades fundamentales, ni sus objetivos vitales, se tiene la ventaja que lo único que no cambia es el objetivo del trabajo. El objetivo no es el trabajo sino la misma acción de formación. Al cambiar el trabajo, la forma del trabajo, cambian las relaciones de producción y de ejecución, manteniendo el problema de las condiciones primarias de la acción de formación. En el inicio de la lección se mantenía el valor dialógico a través del comentario, el estudio del texto sostenía la necesidad de la conversación como matriz esencial de la interacción humana. Hemos adquirido la cultura del libro manteniendo la necesidad básica de la comunicación personal; ahora tenemos la necesidad de sostener la conversación, la cultura lectoescritora y asimilar culturalmente la mediación de las TIC: es el proyecto de sostener los ideales de Paideia en este nuevo contexto, como cuando se crean las universidades hace 1000 años.

A esa acción de formación se suman dos sistemas tecnológicos no siempre gremialmente bien avenidos: la tecnología del ordenador y la tecnología de los sistemas de telecomunicación. El ejemplo más evidente de los primero se encuentra en los programas de formación asistidos por ordenador y del segundo las teleconferencias.

El concepto de "aula virtual", cuando lo acuña Roxanne Hiltz [13] lo hace pensando en un analogado electrónico de las actividades que se realizan en el aula, incluyendo "discusiones, conferencias y exámenes". En ese concepto se funden las posibilidades de los dos tipos de tecnologías, cuyas ejemplificaciones más relevantes se encuentran en el hipertexto que proporciona el ordenador y la posibilidad de comunicación a distancia con tutores o entre iguales.

La demostración de la virtualidad del empleo de las TIC en las acciones de formación no está en el empleo en cuanto tal, sino en la demostración de que determinadas posibilidades de las acciones de formación nunca serían reales sin el empleo de las TIC. Este es el reto pedagógico de las TIC en el diseño de las acciones de formación.

\section{(a) Componentes esenciales en un espacio real de formación}

Denominaremos real los componentes primarios, independientes del espacio cultural considerado, que caracterizan la forma del proceso formativo en la especie, donde la modulación del comportamiento en el tiempo se encuentra mediado socialmente. La cultura 
objetiva hace referencia al aspecto de patrimonio de productos culturales y de oportunidad cultural, la cultura subjetiva al proceso de mediación social mediante el cual el sujeto accede o incorpora, actualiza, su oportunidad en el patrimonio.

En una perspectiva vygotskiana, y sin el ánimo de exhaustividad, ese espacio real se compone de dos actores en relación asimétrica: (i) el actor aprendiz, (ii) la agencia de formación . (iii) El tercer componente es el contenido de la acción de formación (segmento de cultura objetiva, o "problema"). El (iv) es el estado del actor en la situación propuesta por la acción de formación, la cual define la ZDP del aprendiz, conocimiento previo necesario. La relación entre la propuesta formativa y la cultura define su condición de potencialmente significativa y la relación al estado del actor su condición de psicológicamente significativa, la disposición de la secuencia de actividad define su condición de pedagógicamente adecuada.

\section{(b) Circunstancias del aprendiz y TIC}

El término circunstancias lo empleamos en el sentido de reconsiderar los aspectos fundamentales que contiene la situación de enseñanza aprendizaje y las transformaciones que son susceptibles de ser introducidas desde las TIC respecto a entornos en los que se introdujeron los procesos lectoescritores, dentro de situaciones en los que imprescindiblemente estaba directa o indirectamente presente la comunicación oral.

Begoña Gros y sus colaboradores, con buen criterio aluden a los grandes aspectos de esa situación y a las grandes categorías de productos TIC que pueden ser diseñados: "tutorial", práctica y aplicaciones, simulaciones, hipertextos-hipermedias [14].

(i)- Los apuntes de los estudiantes en las clases demuestran su necesidad subjetiva de preservar la información aprovechando la eficacia de soportes materiales (tecnología del texto), conciencia implícita de las limitaciones de almacenamiento de la memoria y limitaciones de la mediación de oralidad. De lo primero ya dio muestra científica Ebbinghaus. El alumno selecciona del contexto informativo de oralidad los elementos que considera pertinentes y construye por la escritura un depósito artificial: se plantea el problema de la fidelidad de transcripción, suficiencia de lo transcrito, utilidad en la recuperación del contenido, adecuación a su particular ZDP... A todo ello hay que añadir que el tamaño del grupo posibilita-imposibilita la contrastación personalizada de la recepción de información y la comprobación de la fidelidad de lo transcrito. Junto a ello, la exigencia de presencia para que el proceso tenga lugar.

(ii)- Se completa con referencia a textos y bibliografía. Si se valorara la construcción de materiales en soporte escrito que los alumnos universitarios pueden disponer, construido especialmente con el objetivo de favorecer la enseñanza con éxito en el aula, se comprobaría la escasa utilización del recurso. En el fondo cuenta también el hecho de que el investigador, ni personal ni institucionalmente, considera que la elaboración de estos materiales guiados en su composición por el propósito de orientar el aprendizaje (divulgación científica) constituya un propósito esencial para el desarrollo del conocimiento.

Con estas dos sencillas referencias se puede explicitar el bajo nivel de utilidad real respecto a objetivo de la comunicación oral en el aula, y del bajo nivel de aprovechamiento del espacio virtual de comunicación que introduce el soporte escrito en el contexto de aula. El sistema convencional de aula trabaja con memoria humana y memoria externa muy imperfecta (apuntes) y una memoria difusa (referencias textuales o bibliográficas) de organizaciónrecuperación muy imperfecta. Como se ve intentamos mostrar que el problema de las TIC no es el de solventar o favorecer distancias, sino el de favorecer el funcionamiento del aparato mental con los recursos disponibles. La reflexión debe orientarse por el análisis de las funciones de transmisión, almacenamiento y recuperación de la información, más que por la vía de la demostración de la posibilidad de un aprendizaje a "distancia". 
(iii)- Comprensión es el término clave en el discurso de los profesionales sobre enseñanzaaprendizaje y es también el término clave en las relaciones comunicativas entre personas en la vida ordinaria. Tanto en el aula como en la situación vital la comprensión aparece como el término que refleja la meta final del proceso de interacción y el fundamento para toda ulterior toma de decisiones.

Los profesores buscan indicios para valorar que la comprensión ha tenido lugar y los alumnos cifran en ella la utilidad de la situación y la medida de su propia capacidad en la tarea. Entre esos indicios se encuentran: la posibilidad de resumir, sin alterar en lo fundamental, una secuencia larga de informaciones; parafrasear o recomponer tal secuencia con otros materiales expresivos pero manteniendo homología entre el contenido; contestar correctamente preguntas acerca de componentes del contenido o aplicar reglas de transformación de componentes a datos diferentes a los que se emplearon en anteriores aplicaciones ; actuar siguiendo procedimientos y aplicaciones que demuestran haber comprendido instrucciones o reelaborar el contenido obteniendo consecuencias. En todos los casos parece existir un contrato implícito donde uno actúa siguiendo la lectura de las expectativas del otro. Más adelante nos referiremos a esta lectura y concierto lectura de la mente o teoría de la mente.

En todos los casos de testimonios de comprensión el Actor que comprende va más allá de las palabras o de las acciones puntuales. P.e. si se nos comunica que el 747 de Madrid a Lisboa se ha estrellado, la comprensión de la noticia supone reconstruir toda la situación de referencia a partir de elementos que se contienen en la estructura cognitiva donde 747 alude a avión, Madrid-Lisboa es una ruta aérea...; de análoga manera si entramos en un bar y se acerca una señorita con la carta de menús, tan sólo por este hecho comprendemos que en la situación del restaurante, ella es una camarera que nos ofrece la selección de la comida, con los precios indicados...etc. Tales reconstrucciones que permiten alcanzar el sentido de una palabra (como los pronombres en un discurso) o de una acción (como en las dos situaciones del ejemplo) se denominan procesos anafóricos. No hay nada en las palabras o en el movimiento de la camarera que lleve a la anáfora de la situación donde las inserta el interlocutor; el proceso se pone en marcha rápidamente tan pronto como los elementos del discurso o de la situación son procesados por el sujeto recurriendo a los materiales de significación que tiene almacenados.

Los elementos que se incorporan a esos patrones previos de expectativas se procesan más rápidamente y los que las transgreden producen desconcierto y demora. Las palabras o las acciones activan los procesos mentales trayendo a colación inferencias, presupuestos, expectativas...Todo este proceso guía la interpretación y constituye el propio mensaje comprendido [15]. Esos procesos anafóricos los realiza el estudiante si puede en solitario o en comentario entre iguales, pero ni los apuntes, ni a veces los textos aludidos prevén estas necesidades de comprensión.

El proceso fundamental de la comprensión siempre se lleva a cabo en función de algo que ya se conoce. De tal forma que la propuesta de conocimiento nuevo se asocia a un proceso de relación con elementos ya conocidos, lo que supone: la existencia de urdimbres de datos almacenados y procedimientos para la creación de nuevas relaciones entre los nuevos significados y aquellas estructuras almacenadas. La comprensión de tal relación en el actor que informa es lo que permite estructurar los nuevos mensajes de tal manera que sea potencialmente significativos para el receptor. La existencia de tales procesos es el fundamento básico de cuanto denominamos pedagogía: la habilidad de disponer los mensajes de manera que aseguren la comprensión, para un sujeto determinado en una determinada situación. La configuración de esos recursos de la habilidad no solamente se rige por criterios lógicos, sino incluso estéticos; es técnica y es arte; el criterio-fundamento por el que se lleva a cabo la configuración de la propuesta (enseñanza) se compone de materiales muy 
heterogéneos, desde conocimientos científicos de los que se extraen inferencias para la acción, experiencias anteriores, teorías personales, ... ; todos esos materiales componen el criterio pedagógico de intervención del actor docente o de la situación de la que extrae los significados el Actor que comprende.

El concepto de tutoría en es el fondo el mecanismo de favorecimiento de la comprensión que crea la institución y contiene el rol profesional. Está mediado por la palabra y se orienta hacia el texto.

\section{(iv) La construcción de entornos hipertextuales.}

La cibernética proporciona, en sí misma, un sistema renovado y reasuntivo de semiotización y de representación, el cual añade en su propia estructura -además de aquello que ya poseían el lenguaje y la escritura- una función de simulación de la propia operación de conocimiento. Esta noción de simulación no tiene nada que ver con la idea discutida del ordenador como metáfora de la mente. El texto proporcionaba argumentación proposicional y representación (imagen, grafos, esquemas); la hipertextualidad puede introducir, además, la animación, el vídeo y la palabra.

\section{(c) Circunstancias del docente}

La opinión más generalmente asumida en el proceso de enseñanza aprendizaje es que la condición necesaria para enseñar es saber. De ahí que no se discuta la asociación entre investigación y enseñanza. Ese saber es propiedad personal del que sabe y no puede ser suplantada vicariamente por nadie. Pero este principio es válido únicamente en los contextos de oralidad y de presencialidad de la comunicación. Precisamente el texto invalidó el principio, introduciendo otro virtual de que los libros enseñan en ausencia de su autor. Autores escribas pueden colaborar escribiendo contenidos diferentes, porque la competencia necesaria (escritura) es compartida en comparables grados de excelencia.

Un problema nuevo que introducen las TIC es que la fabricación de productos guiados por objetivos de formación requieren competencias difícilmente acaparables entre los compositores de hipertextos u otros materiales y servicios pedagógicos. La necesidad de equipos interdisciplinares hace que en los contextos virtuales de las TIC convierte en impedimento la actual configuración de equipos docentes, la actual adscripción de profesores a Centros, el actual catálogo de plazas docentes. La construcción de los objetos-TIC requiere la reflexión del proceso pedagógico al que pretenden servir, ya que el uso está condicionado por el diseño y los contenidos-TIC requieren de la elaboración acorde a las posibilidades del soporte. Por no entrar más que en Facultades Universitarias españolas, generalmente de Ciencias, la guía pedagógica implícita de estructuración de los textos por exigencias de conectividad en los hipertextos promueve la comprensión del producto final; pero mientras en las Facultades de Educación no se promuevan estos equipos interdisciplinares no se crearán los contextos adecuados para el desarrollo de pensamiento pedagógico en el contexto de las TIC. No se convierte la Pedagogía en campo de conocimiento interlocutor en el contexto cultural de las TIC. Si la Pedagogía se aísla de las TIC, reducirá su importancia a la reflexión, importante por otro lado, de las necesidades educativas primarias, las cuales se mantienen como necesidades básicas de todo contexto cultural. Saber cómo debiera ser hecho, sin hacerlo nunca, no es una meta aceptable para el pensamiento pedagógico, sobre todo cuando ha privilegiado epistémicamente el marco de la reflexión en la acción. Esta reflexión nunca será adecuada si, siempre, es reflexión de uno sobre la acción de otro. La enseñanza de las TIC se reduciría a contar lo que otros están haciendo y no a contar la reflexión sobre lo que se hace. Desde este punto de vista deben reconsiderarse, en el justo valor que ya tienen y en el que han de adquirir los movimientos de renovación pedagógica. 


\section{Procesos algorítmicos y procesos heurísticos}

No todo en la actividad mental depende del estado de cosas de la estructura, también la situación planteada posee calidades de adecuación- inadecuación, las cuales crean oportunidad para el sujeto; esas calidades son las que configuran lo que denominamos situación potencialmente significativa para un sujeto. Ese potencial dice relación al mismo tiempo a los límites de la destreza y a limitaciones de los datos respecto al sujeto.

Los problemas en la vida diaria se pueden agrupar en dos categorías: estructurados respecto a reglas de resolución y no estructurados. Los primeros son los problemas típicos de los ejercicios escolares, donde la regla o principio es presentado previamente, y la solución depende ordinariamente de aplicación de un algoritmo. A los problemas no estructurados respecto a regla conocida, para no perdernos en eternas cavilaciones, les aplicamos procedimientos heurísticos. Los educadores generalmente afrontan problemas, sin regla previa conocida, en unos casos representan dilemas de acción. En la escuela ocupan un lugar importante los procesos algorítmicos en las tareas, pero en la vida real es suficiente para permitir la convivencia, la interacción entre personas, y el modo de vida los procesos heurísticos.

En el contexto comunicacional de aula, la actividad que generalmente se enseña es la de los problemas ejemplares y las formas ejemplares de resolución que enseña el profesor. En el contexto virtual que propone el texto, los ejercicios que acompañan (unos resueltos, otros por resolver, otros con solución final sin proceso...). El alumno camina por esa vía de actividad generalmente sólo o con el auxilio de iguales.

Las TIC permiten crear un contexto virtual de actividad configurada, p.e. por una Base de Datos, (Objeto), susceptible de contener problemas de cualquier categoría, con posibilidades de ejercicios modelos, auxilios de procedimiento, herramientas, referencias a fórmulas o contenidos...Mientras hemos visto muchos ejemplos de hipertextos, no sabemos si existen Objetos para este servicio. Las herramientas para fabricarlos son accesibles.

El campo de la formación permanente, la formación inicial del profesorado es inmediatamente perfeccionable virtualmente mediante tutoriales de los que hay muchos ejemplos en la literatura.

\section{(e) Circunstancias del conocimiento del sujeto en el problema}

Los preconceptos y, en general las limitaciones de entrada, de los aprendices en espacio de aula son condicionantes del rendimiento y compone habitualmente el lamento de los profesores porque atender también este aspecto limitaría gravemente las posibilidades de desarrollo del programa establecido. Las posibilidades de remedio de esta situación desde el texto, son muy limitadas. Volver a estudiar lo necesario es un proyecto irreal porque se carece de criterio sobre cómo y dónde. Pero esta situación puede ser perfectamente auxiliable desde el hipertexto.

Quizás una de las aportaciones más interesantes de las TIC es la posibilidad de horizontalización del proceso de transmisión de conocimientos. Clásicamente el flujo posible de comunicación docente-discente estaba direccionado del primero al segundo. Las aportaciones de las listas de distribución democratizan el sistema de manera que todos los participantes se convierten a la vez en posibles docentes y discentes, con aportaciones de diverso grado de profundidad, acierto y punto de vista y las información se transmite por igual a todos los miembros que participan en esta lista. De esta manera el proceso de transmisión de la información es más abierto y no sujeto a las normas jerárquicas en la que muchas veces está sumido el proceso educativo [16]. 


\section{(f) La transformación ecológica de la actividad mental}

Se han escrito bastantes libros y ensayos sobre la transformación ecológica del entorno para la actividad humana en general como consecuencia de las TIC [17]. Frecuentemente, como en el caso de Javier Echeverría, aluden a la virtualización del espacio vital mediante alusiones a "dinero virtual", "calles virtuales"... Tiene el riesgo este proceso imaginativo de llevar al lector a un mundo sin calles, sin dinero, sin plazas; cuando, en rigor, sigue habiendo intercambio, calles, plazas, sujetos con rostro, sin rostro y miedo a la mala catadura. La situación tenemos que interpretarla en el sentido de que al espacio real se añade otro virtual, configurando entre ambos el espacio vital de los individuos. Las consecuencias de esta situación empezamos meramente a vislumbrarlas.

En la cultura escriba los niños "van al colegio", entran en el entorno institucional en buena medida virtual, con entidad diferente al mundo real (recuérdese que real no se contrapone a imaginario, sino a posible, y la escuela es un mundo posible gracias a la escritura que deviene actual). Ese mundo escriba tiene "guías especializados": profesores, pedagogos, psicopedagogos, escritores de textos "adecuados", planes de enseñanza, currícula, exámenes, bibliotecas especializadas...

El mundo real sigue sus propios derroteros y en él la trama de interacciones, la urdimbre comunicacional, los procesos de identificación, la historia emocional, los encuentros fortuitos, el viaje, los grupos de iguales... las corrientes de opinión... y otros mediadores sociales de la comunicación procedentes de interfaces de la técnica de reproducción, operan eficazmente configurando opiniones, actitudes, valores, encuentros interculturales... etc. Pero esos mediadores sociales de la comunicación oral y escrita, tienen una homología de acción, que permite la transferencia de habilidades. Bien que mal aprenden a interpretar, a guiarse en las bibliotecas, hay plan y orientación real y posible, porque hay sitios para ello.

En los entornos que se recrean con las TIC se radicaliza la soledad del actor frente a la actividad posible. Pero no hay guías, la orientación es deficiente, se carece de plan, no hay tutoría porque no hay tutores: es un espacio cada vez más lleno, más amplio, más complejo, pero sin pautas, ni otro plan que el de seguir creciendo. Los usos planificados en este espacio se aprenden en los puestos de trabajo, en ocupaciones específicas (p.e. la banca, la investigación...), en aquellas instituciones en las que las TIC se encuentran insertadas en la ejecución de sus proyectos. Fuera de esos ámbitos los sujetos se encuentran masivamente expuestos a las utilidades mentales de muchas de las tecnologías (p.e. T.V.) pero sin las actitudes necesarias para la interpretación, la crítica, la selección...

El pensamiento pedagógico, respecto a la educación informal, proyecta, muchas veces, sus preocupaciones "formales", pero no parece ocupada en desentrañar la trama formativa de ese espacio real, ni en el vector conversacional (oralidad), ni en el vector (medios de comunicación), ni parece demasiado volcado a investigar el vector TIC. Ese espacio real entreverado de mediaciones sociales de todo tipo no compone la troncalidad de los estudios sobre la educación; más bien se asocia al aparato psíquico a su desarrollo y a la actividad mental asociada al currículo (conocimiento por el texto). Mientras tanto, la gente se sorprende que la familia sea un entorno muy importante de anidamiento de la violencia maligna (malos tratos), que la infancia y la adolescencia padezcan más agresión de la sospechada, que los desórdenes emocionales (ansiedad, depresión, angustia...) estén más extendidos de lo que estamos dispuestos a admitir... La Pedagogía sabe poco, de manera contrastada, de lo que está pasando.

Porque muchas de las mediaciones tecnológicas se encuentran sueltas y a su aire (los adultos son tan novatos como los niños) quienes entran en esas mediaciones quedando absortos pueden parecer entontecidos y se atribuye el atontamiento al cacharro. Dice Sartori: "...el 
punto central de mi discurso: el hecho de que la televisión modifica radicalmente y empobrece el aparato cognoscitivo del homo sapiens... el teleespectador es más un animal vidente que un animal simbólico... el hecho de ver lo acerca a sus capacidades ancestrales" [18]. Para poderlo justificar necesita acudir a un principio de funcionamiento mental falso: "la imagen se ve y eso es suficiente". Para comprender la imagen, la palabra o el texto, ya lo hemos indicado, comprensión implica relación a otros elementos de la memoria, exige la actividad del sujeto. Otra cosa es la atribución de credibilidad o la disposición del material televisivo para que sea potencialmente significativo para una audiencia masiva. Al tiempo que se produce la queja no se ha dado suficiente importancia a la divulgación científica como compromiso fundamental de la actividad científica. En la cultura escrita hay conformidad sobre el hecho de que es "básico" el aprendizaje instrumental. Hoy los instrumentos de la comunicación han aumentado. Sartori también afirma que "la TV es también, a la vez, paideia, un instrumento antropogenético, un medio que genera un nuevo anthropos, un nuevo tipo de ser humano". Es verdad lo de instrumento, no lo es lo del nuevo tipo, sino como metáfora: es parte del nuevo contexto en el que tenemos que ser humanos. Esto define una tarea cultural no un pecado original. Es un error de bulto considerar la TV como un instrumento de imágenes; aunque las imágenes constituyen otras formas de hablar, la TV es un instrumento de la comunicación audio-visual. Falla, pues, la reflexión por su base. Estamos ante, como en el caso de libro, problemas de uso, problemas de contenido, problemas de actitud...problemas de formación. La pregunta es ¿Qué pasa y qué hacer en este espacio real (no institucional) donde están esas mediaciones y qué ha de pasar con las instituciones y los roles de la formación, qué ha de pasar con los sistemas de formación? El libro instituyó el currículo, ¿qué debe ser instituido cuando en el contexto están las TIC?.

\section{Bibliografía}

BARROSO JEREZ, C (1997): Ciencia, Tecnología y Educación. Gobierno de Canarias, La Laguna.

BENEDICT, R. (1989 v.o. 1934): El hombre y la cultura . Ed. Edhasa. Barcelona.

BLOOM, P.(1997):El cierre de la mente humana. Plaza y Janés. Barcelona.

CASTELLS, M. (1998): La societé en réseaux. L’ère de l'information. Fayard. París.

DAHL, S.(1997 v.o. 1927): Historia del Libro . Altaya. Barcelona.

DELEUZE, G. (1968) : Differencia y répétition. PUF. París.

DUCROCQ, A. (1993) :Le changement global. Ed. Jean Claude Lattés. París.

ECHEVERRÍA, J. (1994): Telépolis. Destino. Barcelona.

Id. (1995): Telepolitas domésticos. Anagrama. Barcelona.

FERRAROTTI, F. (1997): La perfezione del nulla. Premesse e problemi della rivoluzione digitale. Laterza. Roma-Bari.

GARCIA CARRASCO,J.-GARCÍA DEL (1999): Teoría de la Educación, II volumen. Ediciones Universidad de Salamanca. Salamanca.

GERGEN, K. (1992): El Yo saturado. Dilemas de identidad en el mundo contemporáneo. Paidós. Barcelona.

GOODY, J. (1990): La lógica de la escritura y la organización de la Sociedad. Alianza. 
Madrid.

GROS, B.(1997): Diseños y programas educativos. Pautas pedagógicas para la elaboración de software. Ariel. Barcelona.

LEVY, P. (1988) : Qu'est-ce que le virtuel. La Découverte. París.

LEVY, P. (1990): Les tecnologies de l'intelligence. L'avenir de la pensée à l'ère informatique. La Découverte. París.

NEGROPONTE,N. (1995): El mundo digital. Editorial B, Barcelona.

LATOUCHE, S. (1991): El planeta de los náufragos.Ensayo sobre el posdesarrollo. Acento. Madrid.

LEROI-GOURHAN,A. (1964) : Le geste et la parole, 2 Vol. Albin Michel. París.

RHEINGOLD,H. (1993) : La Réalité virtuelle. Dunod. París.

ROSNAY,J. (1995): L’homme symbiotique. Seuil, París.

SANFORD,A. (1990): La mente del hombre. Alianza. Madrid.

SARTORI, G. (1998):Homo videns. La sociedad teledirigida. Taurus. Madrid

SERRES,M. (1994): Atlas. Julliard. París.

TIFFIN,J.-RAJASINGHAN,L. (1997): En busca de la clase virtual. La educación en la sociedad de la información. Paidós. Barcelona.

\section{NOTAS}

[1] Dahl, Svend (1997 v.o. 1927) Historia del Libro (Ed.Altaya, Barcelona) ."... hay razones para creer que la historia del libro no acabará con el fin del siglo XX. No es puro azar que se luche empeñadamente contra el analfabetismo allí donde todavía domina y que el libro desempeñe un papel principal en las tareas culturales de la UNESCO. Habrá siempre una misión para este práctico medio de comunicación que posee la ventaja esencial sobre todos los demás de no ser pasajero con ellos, sino un perdurable depósito de pensamientos y saberes, acciones, sentimientos y fantasías de la humanidad, siempre dispuesto a abrirse de nuevo." p.292.

[2] Leroi-Gourhan, André (1964) Le geste et la parole, 2 Vol. (Ed. Albin Michel, París).

[3] Este planteamiento se desarrolla con amplitud y mayor documentación en García Carrasco, J. - García del Dujo, A. (1999) Teoría de la Educación, II volumen (Ediciones Universidad de Salamanca) (en prensa)

[4] Goody, Jack (1990) La lógica de la escritura y la organización de la Sociedad (Ed. Alianza, Madrid)

[5] Barroso Jerez, Clara (1997) Ciencia , Tecnología y Educación (Ed. Gobierno de Canarias)

[6] Benedict, Ruth (1989 v.o. 1934) El hombre y la cultura (Ed. Edhasa, Barcelona)."La 
costumbre tradicional, refiriéndonos al mundo, es una masa de conducta detallada más sorprendente que la que cualquier persona pueda desarrollar jamás en acciones individuales, por anómalas que sean"..."ningún hombre mira jamás el mundo con ojos prístinos".(p.14)

[7] Castells, Manuel (1998) La societé en réseaux. L’ère de l'information (Ed. Fayard, París) pp.86 y ss.

[8] Deleuze, Gilles (1968) Differencia y répétition (Ed. PUF., París)

[9] Lévy, Pierre (1988) Qu’est-ce que le virtuel (Ed. La Découverte, Paris) p.15-16.

[10] Serres,Michel (1994) Atlas(Ed. Julliard, París)

[11] Howard, Rheingold (1993) La Réalité virtuelle(Ed. Dunod, París)

[12] Lambert Steve , Ropiequet Suzanne (1986) CD-ROM, the New Papyrus (Microsoft Press, Redmond, WA), contiene el texto de Bush, As we may Think.

[13] Tiffin,John- Lalita Rajasinghan (1997) En busca de la clase virtual. La educación en la sociedad de la información. (Ed. Paidós, Barcelona)

[14] Gros, Begoña (1997) Diseños y programas educativos. Pautas pedagógicas para la elaboración de software (Ed. Ariel, Barcelona) p.17.

[15] Sanford, Anthony J. (1990) La mente del hombre (Ed. Alianza, Madrid) p. 40 y ss.

[16] Una relación de las listas de distribución residentes en el servidor de la Red Iris se puede consultar en la dirección: http://www.rediris.es/list/buscon.es. Entre ellas podemos destacar las siguientes relativas al campos de la educación:

- AIDIPE-L, centrada en Metodología de investigación, Medición y Evaluación, Orientación escolar y profesional, Diagnóstico educativo y Pedagogía de la diferencia.

- ALFABETO: que pretende constituirse en el foro de encuentro de los profesionales dedicados a la investigación, enseñanza y promoción de la lectura y la escritura como procesos pedagógicos, psicológicos, lingüísticos y sociológicos.

- ECONEDUC: centada en la Economía de la Educación.

- EDUTEC-L: es una lista de discusión para tratar temas exclusivamente académicos referentes a la Tecnología Educativa y Nuevas Tecnologías aplicadas a la Educación de ámbito universitario español e iberoamericano.

- EDULIST: Pretende ser un foro de intercambio, discusión y libre circulación de la información entre los docentes y no docentes en cualquier punto de la Red y en cualquier lengua.

- EDUADULTOS: centrada en el campo de la Educación de Adultos y asentada sobre el diálogo crítico y reflexivo de todos sus participantes. Su riqueza principal no estará en el intercambio simple de ideas y propuestas sino en el diálogo progresivo sobre ellas.

- ETNOEDU: la iniciativa de esta lista surge a propuesta de la recientemente constituida red europea de Etnografía de la Educación y trata de sistematizar el "corpus científico" producido por la investigación etnográfica en el ámbito educativo.

- EVALATEI: que es un foro sobre Televisión Educativa Iberoamericana. 
- EVALUNIV: centrada en la mejora de la calidad y la evaluación de las universidades.

- EDUFIS: sobre Educación Física.

- FILONINOS: cuyo tema básico es la Filosofía para niños.

- GACELA: cuyo objetivo es la formación permanente del profesorado en el área de Ciencias.

- PAIDEIUS: sobre innovación didáctica en Derecho.

- PSICOEDUC: el propósito de servir de foro de debate e intercambio de ideas entre todas las personas de habla hispana interesadas en el estudio y en la intervención en el campo educativo desde una perspectiva psicológica.

Información sobre como darse de alta y trabajar en estas listas se puede encontrar en la dirección de Internet indicada previamente.

[17] Lévy, Pierre (1990) Les tecnologies de l'intelligence. L'avenir de la pensée à l'ère informatique (Ed. La Découverte, París), Lambert Steve, Ropiequet Suzanne (1986)CDROM, the New Papyrus (Microsoft Press, Redmond, WA), contiene el texto de Bush ,As we may Think. Callon, Michel (1989) La Science et ses réseaux. Genèse et circulation de faits scientifiques (La Découverte - C.E. - UNESCO. París-Strasbourg), Echeverría, Javier (1994) Telépolis (Ed. Destino, Barcelona), Id. (1995) Telepolitas domésticos(Ed. Anagrama, Barcelona). Rosnay, Joël de (1995) L’homme symbiotique (Ed. Seuil, París). Ducrocq,albert (1993) Le changement global(Ed. Jean Claude Lattés). Latouche,Serge (1991) El planeta de los náufragos.Ensayo sobre el posdesarrollo (Ed. Acento,Madrid). Gergen, Kenneth J. (1992) El Yo saturado. Dilemas de identidad en el mundo contemporáneo, (Ed. Paidós, Barcelona)

[18] Sartori, Giovanni (1998)Homo videns. La socidad teledirigida (Ed. Taurus, Madrid) p.17 y 32. "La TV invierte la evolución de los sensible en inteligible y lo convierte en el ictu oculi, en un regreso al puro y simple acto ver. La TV produce imágenes y anula conceptos, y de este modo atrofia nuestra capacidad de abstracción y con ella toca nuestra capacidad de comprender" (p.46). Ver también Bloom, Pierre (1997)El cierre de la mente humana (Ed. Plaza y Janés, Barcelona). Ferrarotti, Franco (1997)La perfezione del nulla. Premesse e problemi della rivoluzione digitale (Ed. Laterza, Roma-Bari). Negroponte, Nicholas (1995) El mundo digital (Ed. B, Barcelona)

(C) Ediciones Universidad de Salamanca. 